\title{
ON GENERAL HIRATA-AZUMAYA GALOIS EXTENSIONS
}

\section{LIANYONG XUE}

Department of Mathematics

Bradley University

Peoria, Illinois 61625

USA

e-mail: lxue@bradley.edu

\begin{abstract}
Let $B$ be a general Hirata-Azumaya Galois extension of $B^{G}$ with Galois group $G$. Then several characterizations of such a $B$ are shown, and an equivalent condition is given for such a $B$ satisfying the fundamental theory.
\end{abstract}

\section{Introduction}

Let $B$ be a ring with $1, G$ be a finite automorphism group of $B, C$ be the center of $B$, and $B^{G}=\{b \in B \mid g(b)=b$ for each $g \in G\}$. In [3, 6, 11], the class of central Galois extensions $B$ over $C$ with Galois group $G$ was studied. In $[5,8,14]$, the class of Hirata separable and Galois extensions $B$ of $B^{G}$ with Galois group $G$ was investigated. In [1, 2, 15], the class of Galois extensions $B$ of $B^{G}$ with Galois group $G$ such that $B^{G}$ is an Azumaya $C^{G}$-algebra was explored, such a $B$ is called an Azumaya 2010 Mathematics Subject Classification: 13B05.

Keywords and phrases: separable extensions, Azumaya algebras, Hirata separable extensions, Galois extensions, Hirata-Azumaya Galois extensions.

Received December 28, 2015

(ㄷ) 2016 Scientific Advances Publishers 
Galois extension. Then in [17], the author studied the class of Hirata separable and Azumaya Galois extensions $B$ of $B^{G}$ with Galois group $G$. This is a broader class of Galois extensions than the class of central Galois extensions. Noting that a Galois extension $B$ of $B^{G}$ with Galois group $G$ such that $B^{G}$ is a separable $C^{G}$-algebra is not necessarily an Azumaya Galois extension Galois group $G$. In [13, 16, 18], a Galois extension $B$ of $B^{G}$ with Galois group $G$ such that $B^{G}$ is a separable $C^{G}$-algebra is called a general Azumaya Galois extension of $B^{G}$ with Galois group $G$. The purpose of the present paper is to study the class of Hirata separable and general Azumaya Galois extensions $B$ of $B^{G}$ with Galois group $G$. We shall show several characterizations of such a Galois extension $B$ and give an equivalent condition for such a $B$ satisfying the fundamental theory which generalizes Theorem 3.4 in [12] and Theorem 3.6 in [17].

\section{Basic Definitions and Notations}

Let $B$ be a ring with $1, C$ be the center of $B, G$ be a finite automorphism group of $B, B^{G}$ be the set of elements in $B$ fixed under each element in $G$, and $B * G$ be a skew group ring over $B$ in which the multiplication is given by $g b=g(b) g$ for $b \in B$ and $g \in G$, and $\bar{G}$ be the inner automorphism group of $B * G$ induced by $G$, that is, $\bar{g}(x)=g x g^{-1}$ for each $x \in B * G$ and $g \in G$. We note that $\bar{G}$ restricted to $B$ is $G$.

Let $A$ be a subring of $B$ with the same identity 1 . We call $B$ a separable extension of $A$ if there exist $\left\{a_{i}, b_{i}\right.$ in $B, i=1,2, \ldots, m$ for some integer $m\}$ such that $\sum a_{i} b_{i}=1$, and $\sum b a_{i} \otimes b_{i}=\sum a_{i} \otimes b_{i} b$ for all $b$ in $B$, where $\otimes$ is over $A$, and an Azumaya algebra is a separable extension of its center ([4]). A ring $B$ is called a Hirata separable extension of $A$ if $B \otimes_{A} B$ is isomorphic to a direct summand of a finite direct sum of $B$ as a $B$-bimodule ([8]). 
We call $B$ a Galois extension of $B^{G}$ with Galois group $G$ if there exist elements $\left\{a_{i}, b_{i}\right.$ in $\left.B, i=1,2, \ldots, m\right\}$ for some integer $m$ such that $\sum_{i=1}^{m} a_{i} g\left(b_{i}\right)=\delta_{1, g}$ for each $g \in G$ ([3]). A ring $B$ is called a Galois algebra over $R$ if $B$ is a Galois extension of $R$ such that $R$ is contained in the center $C$ of $B$, and a central Galois algebra over $R$ if $R=C$ ([6]). We call $B$ a Hirata Galois extension of $B^{G}$ with Galois group $G$ if it is a Hirata separable and Galois extension of $B^{G}$ with Galois group $G$ ([8]). A Galois extension $B$ with Galois group $G$ is called an Azumaya Galois extension of $B^{G}$ if $B^{G}$ is an Azumaya $C^{G}$-algebra ([1,2]) and a general Azumaya Galois extension of $B^{G}$ if $B^{G}$ is separable over $C^{G}$ ([16]).

As in [17], $B$ is called a Hirata-Azumaya Galois extension of $B^{G}$ with Galois group $G$ if $B$ is a Hirata separable and an Azumaya Galois extension of $B^{G}$ with Galois group $G$. In this paper, we call $B$ a general Hirata-Azumaya Galois extension of $B^{G}$ with Galois group $G$ if $B$ is a Hirata separable and a general Azumaya Galois extension of $B^{G}$ with Galois group $G$. A Galois extension $B$ of $B^{G}$ with Galois group $G$ is called satisfying the fundamental theory if $\alpha: H \rightarrow B^{H}$ is a one-to-one correspondence between the set of subgroups of $G$ and the set of separable extensions $A$ of $B^{G}$ in $B$ with the inverse map $\alpha^{-1}: A \rightarrow G(A)$, where $G(A)=\{g \in G \mid g(a)=a$ for all $a \in A\}$.

Throughout this paper, we assume that $B$ is a Galois extension of $B^{G}$ with Galois group $G, C$ the center of $B, J_{g}=\{b \in B \mid b x=g(x) b$ for each $x \in B\}$ for a $g \in G$, and for a subring $A$ of $B$ with the same identity 1, $G(A)=\{g \in G \mid g(a)=a$ for all $a \in A\}$ and $V_{B}(A)=\{b \in B \mid a b=b a$ for each $a \in A$ \} the commutator (also called centralizer) subring of $A$ in $B$. 


\section{Main Results}

Keeping the definitions and notations in Section 2, in this section, we shall show several characterizations of a general Hirata-Azumaya Galois extension $B$ of $B^{G}$ with Galois group $G$, and give an equivalent condition for such a $B$ satisfying the fundamental theory. We begin with a very useful property of an Azumaya algebra given by Ikehata.

Lemma 3.1 ([5], Theorem 1). Let $B$ be an Azumaya algebra and $A$ be a subalgebra of $B$. If $B$ is projective as a left $A$-module, then $B$ is a Hirata separable extension of $A$.

Lemma 3.2 ([4], Theorem 3.8, page 55). Let A be a separable algebra and $D$ be a subalgebra of $A$. If $A$ is a left projective $D$-module and $D$ is a direct summand of $A$ as a D-bimodule, then $D$ is a separable subalgebra of $A$.

Theorem 3.3. The following statements are equivalent:

(1) $B$ is a general Hirata-Azumaya Galois extension of $B^{G}$ with Galois group $G$.

(2) $B$ is a general Azumaya Galois extension of $B^{G}$ with Galois group G such that $C=C^{G}$.

(3) $B$ is a Galois extension of $B^{G}$ with Galois group $G$ such that $B$ is an Azumaya $C^{G}$-algebra and the order of $G$ is invertible in $B$.

(4) $B * G$ is a Galois extension of $(B * G)^{\bar{G}}$ with an inner Galois

group $\bar{G}$ induced by $G$ such that $(B * G)^{\bar{G}}$ is a separable $C^{G}$-algebra and $C=C^{G}$.

Proof. (1) $\Rightarrow(2)$ By hypothesis, $B$ is a general Azumaya Galois extension of $B^{G}$ with Galois group $G$. So it suffices to show $C=C^{G}$. In fact, since $B$ is a Hirata separable Galois extension of $B^{G}$, $V_{B}\left(V_{B}\left(B^{G}\right)\right)=B^{G} \quad\left([8]\right.$, Proposition 4-(1)). Hence $C \subset B^{G}$, and so $C=C^{G}$. 
(2) $\Rightarrow$ (1) Since $B$ is a Galois extension of $B^{G}$ with Galois group $G$, $B$ is a separable extension of $B^{G}$ and a left projective $B^{G}$-module. Also, since $B$ is a general Azumaya Galois extension of $B^{G}$ with Galois group $G$ by hypothesis, $B^{G}$ is a separable $C^{G}$-algebra. Hence $B$ is a separable $C^{G}$-algebra by the transitivity property of separable extensions. Noting that $C=C^{G}$ by hypothesis, we conclude that $B$ is an Azumaya $C^{G}$-algebra. Thus, $B^{G}$ is a subalgebra of the Azumaya $C^{G}$-algebra $B$ such that $B$ is left projective over $B^{G}$. Therefore, $B$ is a Hirata separable extension of $B^{G}$ by Lemma 3.1. Hence $B$ is a general Hirata-Azumaya Galois extension of $B^{G}$ with Galois group $G$.

(1) $\Rightarrow$ (3) By the proof of (1) $\Rightarrow(2)$, we have that $C=C^{G}$ and $B$ is an Azumaya $C^{G}$-algebra. Hence, it suffices to show that the order of $G$ is invertible in $B$. In fact, since $B$ is a general Galois extension of $B^{G}$ with Galois group $G, B^{G}$ is a separable $C^{G}$-algebra. Hence $B^{G}$ is a separable subalgebra of the Azumaya algebra $B$. Therefore $V_{B}\left(B^{G}\right)$ is a separable subalgebra of $B$ by the commutator theorem for Azumaya algebras ([4], Theorem 4.3, page 57). Noting that $B$ is a Hirata Galois extension of $B^{G}$ with Galois group $G$, we conclude that the order of $G$ is invertible in $B$ ([8], Proposition 4-(3)).

(3) $\Rightarrow$ (1) Since $B$ is a Galois extension of $B^{G}$ with Galois group $G$, so $B$ is a left projective $B^{G}$-module. Also $B$ is an Azumaya $C^{G}$-algebra by hypotheses, so $B$ is a Hirata separable extension of $B^{G}$ by Lemma 3.1. Thus $B$ is a Hirata Galois extension of $B^{G}$. Now it suffices to show that $B^{G}$ is a separable $C^{G}$-algebra. In fact, since the order of $G$ is invertible in $B, B^{G}$ is a direct summand of $B$ as a $B^{G}$-bimodule. Noting that $B$ is a separable $C^{G}$-algebra and a left projective $B^{G}$-module, we conclude that $B^{G}$ is a separable $C^{G}$-algebra by Lemma 3.2. Thus (1) holds. 
(3) $\Rightarrow$ (4) Since $B$ is a Galois extension of $B^{G}$ with Galois group $G$, $B * G$ is a Galois extension of $(B * G)^{\bar{G}}$ with Galois group $\bar{G}$ with the same Galois system for $B$. Also, by hypotheses, $B$ is an Azumaya $C^{G}$-algebra, that is, $B$ is separable over $C^{G}$ and $C=C^{G}$. Hence, it suffices to show that $(B * G)^{\bar{G}}$ is a separable $C^{G}$-algebra. In fact, since the order of $G$ is invertible in $B, B * G$ is a separable extension of $B$. Hence $B * G$ is a separable $C^{G}$-algebra by the transitivity property of separable extensions. Moreover, since $B * G$ is a Galois extension of $(B * G)^{\bar{G}}$ with an inner Galois group $\bar{G}$ induced by $G, B * G$ is a left projective $(B * G)^{\bar{G}}$-module. Noting that $\bar{G}$ restricted to $B$ is $G$, we have that the order of $\bar{G}$ is equal to the order of $G$. Hence the order of $\bar{G}$ is invertible in $B * G$. Thus $(B * G)^{\bar{G}}$ is a direct summand of $B * G$ as a $(B * G)^{\bar{G}}$-bimodule. Therefore $(B * G)^{\bar{G}}$ is a separable $C^{G}$-algebra by Lemma 3.2.

(4) $\Rightarrow$ (3) Since $B * G$ is a Galois extension of $(B * G)^{\bar{G}}$ with an inner Galois group $\bar{G}$ induced by $G, B$ is a Galois extension of $B^{G}$ with Galois group $G$ ([9], Theorem 3.1). Also, since $B * G$ is a Galois extension of $(B * G)^{\bar{G}}, B * G$ is a separable extension of $(B * G)^{\bar{G}}$. Therefore, $B * G$ is a separable extension of $C^{G}$ by the transitivity property of separable extensions because $(B * G)^{\bar{G}}$ is a separable $C^{G}$-algebra by hypotheses. Moreover, since $B * G$ is a left projective $B$-module and $B$ is a direct summand of $B * G$ as a $B$-bimodule, $B$ is a separable $C^{G}$-algebra by Lemma 3.2. But $C=C^{G}$ by hypotheses, so $B$ is an Azumaya $C^{G}$-algebra. Next, we show that the order of $G$ is invertible in $B$. Noting that $\bar{G}$ restricted to $B$ is $G$, the order of $\bar{G}$ is equal to the order of $G$. Hence, it suffices to show the order of $\bar{G}$ is invertible in $B$. In fact, since 
$B * G$ is a Galois extension of $(B * G)^{\bar{G}}$ with an inner Galois group $\bar{G}$, $B * G$ is a Hirata separable extension of $(B * G)^{\bar{G}}$ ([8], Corollary 3). Hence $B * G$ is a Hirata Galois extension of $(B * G)^{\bar{G}}$ with an inner Galois group $\bar{G}$. Moreover, since $(B * G)^{\bar{G}}$ is a separable $C^{G}$-algebra, $(B * G)^{\bar{G}}$ is a separable $Z$-algebra, where $Z$ is the center of $B * G$ and is contained in $(B * G)^{\bar{G}}$. Noting that $B * G$ is separable over $C^{G}$, which is contained in the center $Z$ of $B * G$, we have that $B * G$ is Azumaya $Z$-algebra. Thus $V_{B * G}\left((B * G)^{\bar{G}}\right)$ is a separable $Z$-algebra by the commutator theorem for Azumaya algebras ([4], Theorem 4.3, page 57). Hence $B * G$ is a Hirata Galois extension of $(B * G)^{\bar{G}}$ such that $V_{B * G}\left((B * G)^{\bar{G}}\right)$ is a separable subalgebra of $B * G$. Therefore, the order of $\bar{G}$ is invertible in $B * G$ ([8], Proposition 4). This completes the proof.

We remark that Theorem $3.3(1) \Rightarrow$ (4) shows that if $B$ is a general Hirata-Azumaya Galois extension with Galois group $G$, then its skew group ring $B * G$ is also a general Hirata-Azumaya Galois extension with an inner Galois group $\bar{G}$ induced by $G$.

Corollary 3.4. Let $B$ be a general Hirata-Azumaya Galois extension of $B^{G}$ with Galois group $G$. Then $B * G$ is a general Hirata-Azumaya Galois extension of $(B * G)^{\bar{G}}$ with an inner Galois group $\bar{G}$ induced by $G$.

Proof. Since $B$ is a general Hirata-Azumaya Galois extension of $B^{G}$ with Galois group $G, B * G$ is a Galois extension of $(B * G)^{\bar{G}}$ with an inner Galois group $\bar{G}$ induced by $G$ such that $(B * G)^{\bar{G}}$ is a separable $C^{G}$-algebra by Theorem $3.3(1) \Rightarrow(4)$. Hence $B * G$ is a Hirata separable extension of $(B * G)^{\bar{G}}$ ([8], Corollary 3). Thus $B * G$ is a Hirata Galois extension of $(B * G)^{\bar{G}}$ such that $(B * G)^{\bar{G}}$ is a separable 
$C^{G}$-algebra. Clearly, the center $Z$ of $B * G$ is contained in $(B * G)^{\bar{G}}$ and $C^{G} \subset Z^{\bar{G}}$. Therefore, $(B * G)^{\bar{G}}$ is a separable $Z^{\bar{G}}$-algebra. Hence $B * G$ is a general Azumaya Galois extension of $(B * G)^{\bar{G}}$ with inner Galois group $\bar{G}$, and so $B * G$ is a general Hirata-Azumaya Galois extension with Galois group $\bar{G}$.

Corollary 3.5. Let $B$ be a Hirata-Azumaya Galois extension of $B^{G}$ with Galois group $G$. Then $B * G$ is a general Hirata-Azumaya Galois extension of $(B * G)^{\bar{G}}$ with an inner Galois group $\bar{G}$ induced by $G$.

We note that in general for a Hirata-Azumaya Galois extension $B$ with Galois group $G, B * G$ is not necessarily a Hirata-Azumaya Galois extension of $(B * G)^{\bar{G}}$ with an inner Galois group $\bar{G}$ induced by $G$.

Theorem 3.6. Let $B$ be a Hirata-Azumaya Galois extension of $B^{G}$ with Galois group $G$. If $G$ is commutative, then $B * G$ is not a HirataAzumaya Galois extension of $(B * G)^{\bar{G}}$ with an inner Galois group $\bar{G}$ induced by $G$.

Proof. Since $B$ is a Hirata-Azumaya Galois extension with Galois group $G, B$ is an Azumaya Galois extension with Galois group $G$. Hence $B * G$ is an Azumaya $C^{G}$-algebra ([2], Theorem 3.1). But $G$ is commutative by hypothesis, and so $C^{G} G$ is contained in the center of $(B * G)^{\bar{G}}$. Therefore $(B * G)^{\bar{G}}$ is not Azumaya over $C^{G}$. Thus $B * G$ is not an Azumaya Galois extension of $(B * G)^{\bar{G}}$ with an inner Galois group $\bar{G}$ induced by $G$, and so $B * G$ is not a Hirata-Azumaya Galois extension of $(B * G)^{\bar{G}}$ with an inner Galois group $\bar{G}$ induced by $G$. 
Next, we show an equivalent condition for a general Hirata-Azumaya Galois extension $B$ satisfying the fundamental theory. The proof is similar to the proof of Theorem 3.6 in [17] but more elaborate and without using the condition that $B^{G}$ is an Azumaya $C^{G}$-algebra. We begin a lemma.

Lemma 3.7. Let B be a general Azumaya Galois extension of $B^{G}$ with Galois group $G$. Then $B^{H}$ is a separable $C^{G}$-algebra for every subgroup $H$ of $G$.

Proof. Since $B$ is a general Azumaya Galois extension of $B^{G}$ with Galois group $G, B^{G}$ is a separable $C^{G}$-algebra. Hence $B$ is a Galois extension of a separable $C^{G}$-algebra with Galois group $G$. Therefore, $B^{H}$ is a separable $C^{G}$-algebra for every subgroup $H$ of $G$ ([7], Proposition 3.1).

Theorem 3.8. Let B be a general Hirata-Azumaya Galois extension of $B^{G}$ with Galois group $G$. Then $B$ satisfies the fundamental theorem if and only if for any separable extension $A$ of $B^{G}$ in $B, V_{B}(A)=\oplus$ $\sum_{g \in G(A)} J_{g}$.

Proof. $(\Rightarrow)$ Let $A$ be a separable extension of $B^{G}$ in $B$. Since $B$ satisfies the fundamental theorem, we have that $A=B^{G(A)}$; and so $V_{B}(A)=V_{B}\left(B^{G(A)}\right)$. But $B$ is a Galois extension of $B^{G(A)}$ with Galois group $G(A)$, so $V_{B}\left(B^{G(A)}\right)=\oplus \sum_{g \in G(A)} J_{g}$ ([6], Proposition 1). Hence $V_{B}(A)=V_{B}\left(B^{G(A)}\right)=\oplus \sum_{g \in G(A)} J_{g}$.

$(\Leftarrow)$ Since $B$ is a Hirata separable Galois extension of $B^{G}$ with Galois group $G, H \rightarrow B^{H}$ is a one-to-one map from the set of subgroups of $G$ to the set of subextensions $A$ of $B^{G}$ in $B$ ([14], Corollary 3.5). Since $B$ a general Azumaya Galois extension of $B^{G}$ with Galois group $G, B^{H}$ is a separable $C^{G}$-algebra by Lemma 3.7 ; and so a separable extension of 
$B^{G}$ in $B$ for every subgroup $H$ of $G$. Hence $H \rightarrow B^{H}$ is a one-to-one map from the set of subgroups of $G$ to the set of separable extensions $A$ of $B^{G}$ in $B$. Therefore, it suffices to show that for any separable extension $A$ of $B^{G}$ in $B, A=B^{G(A)}$ In fact, since $A$ is a separable extension of $B^{G}$ and $B^{G}$ is a separable $C^{G}$-algebra, $A$ is a separable $C^{G}$-algebra. But, by Theorem 3.3, $C=C^{G}$ and $B$ is an Azumaya algebra, so $A$ is a separable subalgebra of the Azumaya algebra $B$ over $C$. Hence $A=V_{B}\left(V_{B}(A)\right)$ by the commutator theorem for Azumaya algebras ([4], Theorem 4.3, page 57). Moreover, since $B$ is a general Azumaya Galois extension with Galois group $G$, for a subgroup $G(A)$ of $G, B^{G(A)}$ is separable over $C^{G}$ by Lemma 3.7, so $B^{G(A)}$ is a separable subalgebra of the Azumaya algebra $B$ over $C$. Therefore $B^{G(A)}=V_{B}\left(V_{B}\left(B^{G(A)}\right)\right)$ by the commutator theorem for Azumaya algebras. Now, since $B$ is a Galois extension of $B^{G(A)}$ with Galois group $G(A), V_{B}\left(B^{G(A)}\right)=\oplus \sum_{g \in G(A)} J_{g}$ ([6], Proposition 1), and by hypothesis, $V_{B}(A)=\oplus \sum_{g \in G(A)} J_{g}$. Thus $V_{B}(A)=\oplus \sum_{g \in G(A)} J_{g}=V_{B}\left(B^{G(A)}\right)$. Hence $A=V_{B}\left(V_{B}(A)\right)=V_{B}\left(V_{B}\left(B^{G(A)}\right)\right)=B^{G(A)}$. This completes the proof.

Noting that a Hirata-Azumaya Galois extension with Galois group $G$ is a general Hirata-Azumaya Galois extension with Galois group $G$, and a central Galois algebra $B$ with Galois group $G$ is a Hirata-Azumaya Galois extension with Galois group $G$. Theorem 3.8 generalizes the characterizations for a Hirata-Azumaya Galois extension in [17] and for a central Galois algebra in [12] satisfying the fundamental theorem.

Corollary 3.9 ([17], Theorem 3.6). Let B be a Hirata-Azumaya Galois extension with Galois group $G$. Then $B$ satisfies the fundamental theorem if and only if for any separable subalgebra $A$ of $B, V_{B}(A)=\oplus$ $\sum_{g \in G(A)} J_{g} \cdot$ 
Corollary 3.10 ([12], Theorem 3.4). Let B be a central Galois algebra with Galois group $G$. Then $B$ satisfies the fundamental theorem if and only if for any separable subalgebra $A$ of $B, V_{B}(A)=\oplus \sum_{g \in G(A)} J_{g}$.

We conclude the present paper with two examples of a Galois extension $B$ with Galois group $G$ to illustrate that (1) $B$ is a general Hirata-Azumaya Galois extension with Galois group $G$, but $B$ is not a Hirata-Azumaya Galois extension with Galois group $G$; (2) $B$ is a Hirata Galois extension of $B^{G}$ with Galois group $G$, but $B$ is not a general Hirata-Azumaya Galois extension with Galois group $G$.

Example 3.11. Let $B=\mathbb{Q}[i, j, k]$, the quaternion algebra over the rational field $\mathbb{Q}$, and $G=\left\{1, g_{i}\right\}$, where $g_{i}(x)=i x i^{-1}$ for all $x \in B$. Then

(1) $B$ is a Galois extension with Galois group $G$ with a Galois system $\left\{1, i, j, k ; \frac{1}{4},-\frac{1}{4} i,-\frac{1}{4} j,-\frac{1}{4} k\right\}$.

(2) Since $G$ is inner induced by the elements $\{1, i\}, B$ is a Hirata separable extension of $B^{G}$ ([8], Corollary 3).

(3) $B^{G}=\mathbb{Q}[i]$ which is a separable $\mathbb{Q}$-algebra, where $\mathbb{Q}$ is the center of $B$ and $\mathbb{Q}^{G}=\mathbb{Q}$. Hence $B$ is a general Azumaya Galois extension with Galois group $G$.

(4) $B$ is a general Hirata-Azumaya Galois extension with Galois group $G$ by (1), (2), and (3).

(5) Since $B^{G}(=\mathbb{Q}[i])$ is not Azumaya over $\mathbb{Q}^{G}, B$ is not a HirataAzumaya Galois extension with Galois group $G$. 
Example 3.12. Let $A=\mathbb{Q}[i, j, k]$, the quaternion algebra over the rational field $\mathbb{Q}, B=\left\{\left(\begin{array}{cc}a_{1} & a_{2} \\ 0 & a_{3}\end{array}\right) \mid a_{1}, a_{2}, a_{3} \in A\right\}$, the ring of all 2 by 2 upper triangle matrices over $A$, and $G=\left\{1, g_{i}, g_{j}, g_{k}\right\}$, where $g_{i}(a)=i a i^{-1}, g_{j}(a)=j a j^{-1}, g_{k}(a)=k a k^{-1}$ for all a in $A$ and $g\left(\begin{array}{cc}a_{1} & a_{2} \\ 0 & a_{3}\end{array}\right)=\left(\begin{array}{cc}g\left(a_{1}\right) & g\left(a_{2}\right) \\ 0 & g\left(a_{3}\right)\end{array}\right)$ for $g \in G$. Then

(1) It is easy to check that the center of $B$ is $\mathbb{Q} I$, where $I$ is the identity 2 by 2 matrix and $B^{G}=\left\{\left(\begin{array}{ll}q_{1} & q_{2} \\ 0 & q_{3}\end{array}\right) \mid q_{1}, q_{2}, q_{3} \in \mathbb{Q}\right\}$, the ring of all 2 by 2 upper triangle matrices over $\mathbb{Q}$.

(2) $B$ is a Galois extension with Galois group $G$ with a Galois system $\left\{I, i I, j I, k I ; \frac{1}{4} I,-\frac{1}{4} i I,-\frac{1}{4} j I,-\frac{1}{4} k I\right\}$.

(3) Since $G$ is inner induced by the elements $\{I, i I, j I, k I\}, B$ is a Hirata separable extension of $B^{G}$ ([8], Corollary 3).

(4) By (2) and (3) $B$ is a Hirata Galois extension of $B^{G}$ with Galois group $G$.

(5) Since $B^{G}=\left\{\left(\begin{array}{cc}q_{1} & q_{2} \\ 0 & q_{3}\end{array}\right) \mid q_{1}, q_{2}, q_{3} \in \mathbb{Q}\right\}$ which is not a separable $\mathbb{Q} I$-algebra ([10], Example 4.3-(8)), $B$ is not general Azumaya Galois extension with Galois group $G$. 


\section{References}

[1] R. Alfaro and G. Szeto, Skew group rings which are Azumaya, Comm. in Algebra 23(6) (1995), 2255-2261.

[2] R. Alfaro and G. Szeto, On Galois extensions of an Azumaya algebra, Comm. in Algebra 25(6) (1997), 1873-1882.

[3] F. R. DeMeyer, Some notes on the general Galois theory of rings, Osaka J. Math. 2 (1965), 117-127.

[4] F. R. DeMeyer and E. Ingraham, Separable Algebras over Commutative Rings, Volume 181, Springer Verlag, Berlin, Heidelberg, New York, 1971.

[5] S. Ikehata, Note on Azumaya algebras and $H$-separable extensions, Math. J. Okayama Univ. 23 (1981), 17-18.

[6] T. Kanzaki, On Galois algebra over a commutative ring, Osaka J. Math. 2 (1965), 309-317.

[7] T. Kanzaki, On Galois extension of rings, Nagoya J. Math. 27 (1966), 43-49.

[8] K. Sugano, On a special type of Galois extensions, Hokkaido J. Math. 9 (1980), 123-128.

[9] G. Szeto and L. Xue, Skew group rings which are Galois, International Journal of Mathematics and Mathematical Sciences 23(4) (1999), 279-283.

[10] G. Szeto and L. Xue, On central commutator Galois extensions of rings, International Journal of Mathematics and Mathematical Sciences 24(5) (2000), 289-294.

[11] G. Szeto and L. Xue, The structure of Galois algebras, Journal of Algebra 237(1) (2001), 238-246.

[12] G. Szeto and L. Xue, On Galois algebras satisfying the fundamental theorem, Communications in Algebra 35(12) (2007), 3979-3985.

[13] G. Szeto and L. Xue, On Galois extensions of a separable algebra, International Mathematical Forum 3(14) (2008), 677-683.

[14] G. Szeto and L. Xue, On Galois extensions with a one-to-one Galois map, International Journal of Algebra 5(17) (2011), 801-807.

[15] L. Xue, On Azumaya Galois extensions, Bull. Malays. Math. Sci. Soc. (2) 35(2A) (2012), 583-589.

[16] L. Xue, On Galois correspondence of a general Azumaya Galois extension, International Journal of Algebra and Statistics 1 (2012), 45-49.

[17] L. Xue, On Hirata-Azumaya Galois extensions, International Mathematical Forum 8(23) (2013), 1103-1110.

[18] L. Xue, On compositions of a Galois extension of a separable algebra, International Journal of Algebra 8(2) (2014), 67-73. 